\section{Prevalência e variáveis associadas à incontinência urinária no terceiro trimestre gestacional}

\section{Prevalence of variables associated with urinary incontinence in the third trimester of pregnancy}

Cinara Sacomori 1

Leonice Böer 2

Fabiana Flores Sperandio 3

Fernando Luiz Cardoso 4

1-4 Centro de Ciências da Saúde e do Esporte. Universidade do Estado de Santa Catarina. Rua Pascoal Simone, 358. Coqueiros. Florianópolis, SC, Brasil. CEP: 88.080-350. E-mail: csacomori@yahoo.com.br

\begin{abstract}
Objectives: to assess the prevalence of urinary incontinence (UI) in the third trimester of gestation and to identify variables associated with UI during this period.

Methods: a cross-sectional study was carried out with 242 pregnant women near to giving birth at the Carmela Dutra Maternity Hospital, in the Brazilian city of Florianópolis. The study covered socio-demographic factors, clinical, and gynecological and obstetric histories and related issues. The evaluation of urinary incontinence was carried out using the Short Form ICIQ. The data were analyzed using the chi-squared test and Mann-Whitney's $U$, with $p<0.05$.

Results: the prevalence of UI in the third trimestre of gestation was of $59.5 \%(n=144)$, and the va-riables associated with this condition during this period were: age $(p<0.019)$, number of pregnancies $(p=0.021)$, marital status (living with partner) $(p=0.032)$, constipation prior to pregnancy ( $p=0.013)$, chronic bronchitis $(p=0.011)$, UI prior to pregnancy $(p=0.013)$ and symptoms of depression $(p=0.049)$.

Conclusions: even with the care available and the spread of measures to prevent UI, it is still highly prevalent, indicating that little importance has been given to prevention.
\end{abstract}

Key words Urinary incontinence, Pregnancy, Prevalence, Risk factors

\section{Resumo}

Objetivos: descrever a prevalência de incontinência urinária (IU) no terceiro trimestre gestacional e verificar quais variáveis estão associadas à IU nesse periodo.

Métodos: consiste em um estudo transversal, realizado com 242 mulheres que se encontravam no puerpério imediato no Hospital e Maternidade Carmela Dutra, da cidade de Florianópolis. Foram avaliados fatores sociodemográficos, história clínica, antecedentes ginecológicos e obstétricos, além de problemas associados. A avaliação das perdas urinárias foi realizada por meio do ICIQ - Short Form. Os dados foram analisados com os testes quiquadrado e U de Mann Whitney, sendo $p<0,05$.

Resultados: observou-se uma prevalência de IU no terceiro trimestre gestacional de 59,5\% $(n=144)$, e as variáveis associadas a tal condição nesse periodo foram: idade $(p<0,019)$, número de gestações $(p=0,021)$, estado marital (viver com parceiro) $(p=0,032)$, constipação antes da gestação $(p=0,013)$, bronquite crônica $(p=0,011)$, IU antes da gestação $(p=0,013)$ e sintomas depressivos $(p=0,049)$.

Conclusões: mesmo diante dos cuidados disponiveis e em expansão para prevenção de IU, sua prevalência durante a gestação ainda é elevada, configurando pouca importância atribuída ao fator preventivo.

Palavras-chave Incontinência urinária, Gestação, Prevalência, Fatores de risco 


\section{Introdução}

A incontinência urinária (IU) é uma condição comum entre as mulheres e caracteriza-se por ser um importante problema de saúde pública, quer por sua elevada prevalência, quer pelo elevado impacto físico, psíquico e social na vida da mulher. ${ }^{1,2}$ A Sociedade Internacional de Continência define a IU como toda perda involuntária de urina. ${ }^{3}$ Essa disfunção acomete de 20 a 50\% da população feminina ao longo da vida. 4

Sua prevalência durante a gravidez varia entre $32-64 \% .2 \mathrm{~A}$ incidência desse problema na gravidez pode ser subestimada, visto que muitas mulheres não procuram tratamento por vergonha. Além disso, muitas vezes julgam ser um sintoma normal da gestação, cujo sintoma desaparecerá com o tempo, o que acaba impactando negativamente na qualidade de vida.5-8

A IU durante a gravidez pode ser atribuída a mudanças hormonais, mudanças do ângulo uretrovesical, aumento da pressão sobre os músculos elevadores e ligamentos, mudanças nos tecidos conjuntivos, incremento do peso corporal materno e peso do útero gravídico, com crescente aumento da pressão sobre a musculatura do assoalho pélvico.6,7 Já no terceiro trimestre gestacional, a alta prevalência de IU tem sido relacionada a alterações mecânicas, como o efeito da pressão do útero gravídico sobre a bexiga, associado ao aumento de sua sensibilidade e à diminuição significativa da capacidade vesical. 9

No Brasil, há poucos trabalhos científicos dedicados à IU no período gestacional, o que dificulta a identificação da população afetada e a comparação dos resultados. Segundo Higa et al.,10 o reduzido número de estudos referentes aos fatores de risco (ou fatores associados) para IU entre as mulheres brasileiras interfere na identificação dos principais fatores que causam a IU nessa população.

Tendo em vista o aumento dos casos de IU e a existência de poucos estudos que traçam um perfil dessa população no período gestacional, a pesquisa teve como objetivo descrever a prevalência da IU no terceiro trimestre gestacional e verificar quais variáveis estão associadas à incontinência urinária nesse período.

\section{Métodos}

Consiste em um estudo transversal cujas medidas relacionadas ao período antes de gestação e no terceiro trimestre gestacional foram retrospectivas.

Participaram do estudo 242 mulheres que se encontravam no puerpério imediato no Hospital e Maternidade Carmela Dutra, da cidade de Florianópolis, no período de agosto de 2011 a março de 2012. O estudo teve aprovação no Comitê de Ética em Pesquisa do Hospital e Maternidade Carmela Dutra (CAE 0010.0.233.269-11).

O critério de inclusão na pesquisa abrangeu mulheres submetidas ao procedimento de parto normal ou cesáreo nesse período. Os critérios de exclusão foram os seguintes: mulheres que apresentavam incontinência urinária de origem neurológica, histórico de câncer no trato geniturinário, déficit cognitivo, doenças neurológicas diagnosticadas, cegueira, analfabetismo, menores de 18 anos e usuárias dependentes de drogas.

Utilizou-se uma ficha com dados sociodemográficos contendo história clínica, antecedentes ginecológicos e obstétricos, hábitos urinários, prática de atividade física (tipo e frequência) e problemas associados (diabetes, asma, bronquite, depressão e tabagismo). Tais dados foram obtidos na consulta ao prontuário hospitalar e/ou através do autorrelato das pacientes.

Para a avaliação das perdas urinárias, foi utilizado o questionário ICIQ - Short Form, traduzido e validado para português por Tamanini et al. $11 \mathrm{em} 2004$. Esse questionário avalia a frequência e a quantidade de perda urinária, a situação de perda de urina e a interferência dessa condição nas atividades de vida diária. Foram consideradas incontinentes as mulheres que referiram qualquer episódio de perda nesse período. Esse questionário foi aplicado no puerpério imediato com perguntas retrospectivas considerando as perdas urinárias no terceiro trimestre gestacional e antes da gestação.

Também foi utilizado um questionário estruturado com critérios relacionados à constipação intestinal, considerando os dois períodos: prégestação e terceiro trimestre gestacional. A constipação foi avaliada de acordo com os critérios sugeridos por Amselem et al.:12 sensação de bloqueio anal durante a defecação; necessidade de uso da facilitação digital para defecação; sensação de evacuação incompleta; passagem de fezes duras; ocorrência de menos de três evacuações por semana; uso regular de laxantes ou utilização de enemas. A constipação foi caracterizada entre as pacientes que apresentaram três ou mais desses critérios.

Para a referida pesquisa, o procedimento de coleta dos dados consistiu em explicar os objetivos e os benefícios da pesquisa à puérpera e solicitar a assinatura do termo de consentimento livre e esclarecido. A coleta de dados ocorreu no próprio leito hospitalar, passadas no mínimo 12 horas de pós- 
parto.

Os dados foram analisados com recursos de estatística descritiva (frequências, porcentagens, média e desvio padrão), além dos testes quiquadrado ou o de Fisher e U de Mann Whitney, uma vez que os dados não seguiram os critérios de normalidade. Adotou-se um $p<0,05$.

\section{Resultados}

Quanto à descrição das características das participantes (Tabela 1), observou-se que a maioria era casada ou vivia em união consensual $(86,8 \%)$, tinha ensino médio completo/incompleto $(60,3 \%)$ e era de etnia caucasiana $(86,4 \%)$.

\section{Tabela 1}

\begin{tabular}{|c|c|c|c|c|c|c|c|}
\hline \multirow[t]{2}{*}{ Características } & \multicolumn{2}{|c|}{ Todas $(\mathrm{N}=242)$} & \multicolumn{2}{|c|}{ Incontinentes $(\mathrm{N}=144)$} & \multicolumn{2}{|c|}{ Continentes $(\mathrm{N}=98)$} & \multirow[t]{2}{*}{$\chi^{2}(p)$} \\
\hline & $\mathrm{n}$ & $\%$ & $\mathrm{n}$ & $\%$ & $n$ & $\%$ & \\
\hline Escolaridade & & & & & & & $\chi^{2}=1,1 \quad(p=0,577)$ \\
\hline Ensino fundamental & 76 & 31,4 & 48 & 33,3 & 28 & 28,6 & \\
\hline Ensino médio & 146 & 60,3 & 83 & 57,6 & 63 & 64,3 & \\
\hline Ensino superior & 20 & 8,3 & 13 & 9,0 & 7 & 7,1 & \\
\hline Estado marital & & & & & & & $\chi^{2}=5,4(p=0,032 *)$ \\
\hline Casada/união consensual & 210 & 86,8 & 131 & 91,0 & 79 & 80,6 & \\
\hline Solteira/separada & 32 & 13,2 & 13 & 9,0 & 19 & 19,4 & \\
\hline Etnia & & & & & & & NS \\
\hline Branca & 209 & 86,4 & 123 & 85,4 & 86 & 87,8 & \\
\hline Negra/mulata & 33 & 13,6 & 21 & 14,6 & 12 & 12,2 & \\
\hline \multicolumn{8}{|l|}{ Problemas associados } \\
\hline Diabetes & 13 & 5,4 & 7 & 4,9 & 6 & 6,1 & NS \\
\hline Bronquite crônica & 18 & 7,4 & 16 & 11,1 & 2 & 2,0 & $\chi^{2}=6,9\left(p=0,011^{*}\right)$ \\
\hline Asma & 7 & 2,9 & 6 & 4,2 & 1 & 1,0 & NS \\
\hline Depressão & 14 & 5,8 & 12 & 8,3 & 2 & 2,0 & $\chi^{2}=4,3(p=0,049 *)$ \\
\hline Tabagismo & 58 & 24,0 & 36 & 25,0 & 22 & 22,4 & NS \\
\hline Constipação antes da gestação & 76 & 31,4 & 54 & 37,5 & 22 & 22,4 & $\chi^{2}=6,1(p=0,013)$ \\
\hline Constipação no $3^{\circ}$ trimestre & 63 & 26,0 & 44 & 30,6 & 19 & 19,4 & NS \\
\hline $\begin{array}{l}\text { Atividade física antes da } \\
\text { gestação }\end{array}$ & 107 & 44,2 & 63 & 43,8 & 44 & 44,9 & NS \\
\hline Atividade física no $3^{\circ}$ trimestre & 78 & 32,2 & 45 & 31,3 & 33 & 33,7 & NS \\
\hline
\end{tabular}

*Corrigido com o teste exato de Fisher; NS = não significativo.

Conforme indicam as Tabelas 1 e 2, houve prevalência de IU no terceiro trimestre de gestação de $59,5 \%(n=144)$, sendo que a frequência de perdas era uma vez por semana ou menos $(21,5 \%)$, duas ou três vezes por semana $(12,4 \%)$, uma vez ao dia $(8,7 \%)$ e diversas vezes ao dia $(17,0 \%)$. A maioria das participantes referiu que as perdas eram em pequena quantidade $(46,0 \%)$. A prevalência de IU antes da gestação foi de $9,5 \%$, com predomínio das perdas em pequena quantidade e uma vez por semana ou menos. As mulheres com perdas urinárias no terceiro trimestre mencionaram as seguintes situações: antes de chegar ao banheiro $(25,6 \%)$, durante tosse ou espirro (40,5\%), enquanto dormiam $(9,1 \%)$, durante a prática de atividades físicas $(16,6 \%)$, enquanto se vestiam após ter urinado $(16,5 \%)$ e sem razão óbvia $(9,5 \%)$.

Dentre as mulheres incontinentes no terceiro trimestre de gestação, 25,7\% afirmaram que as perdas não interferiam na vida diária, ao passo que $41,0 \%$ afirmaram que tal condição interferia de médio a muito (Figura 1). 


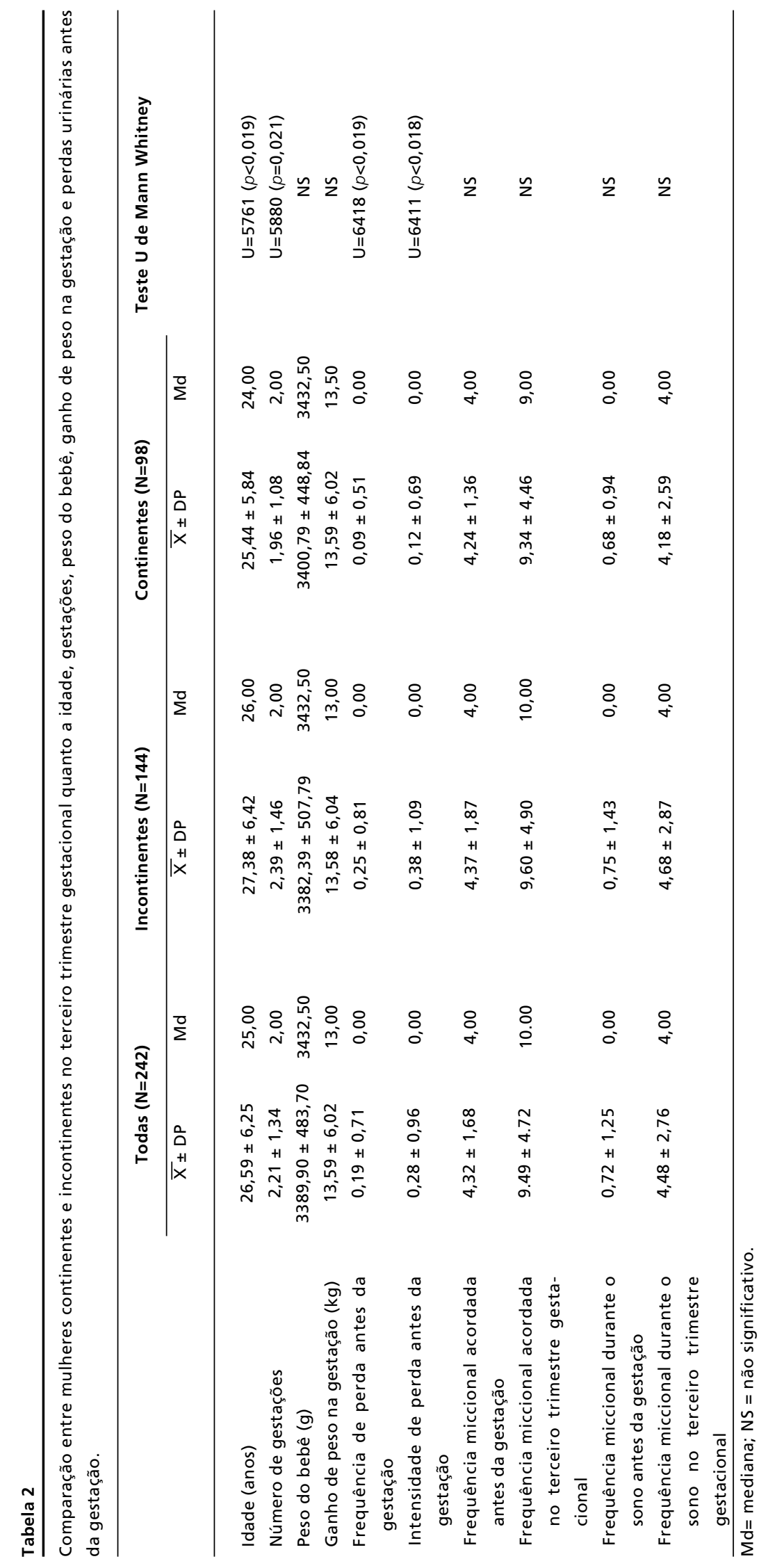


Valores da escala análogo-visual para o impacto da incontinência urinária na qualidade de vida no terceiro trimestre gestacional.

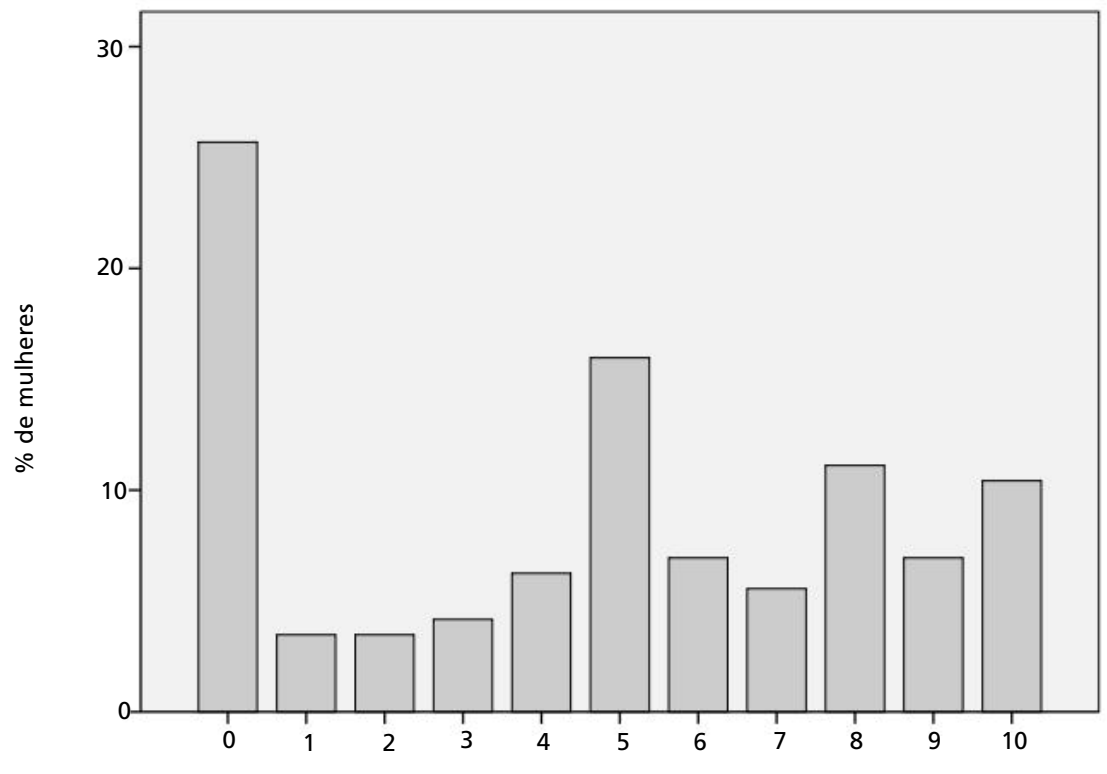

Impacto da incontinência urinária na qualidade de vida no III trimestre

Observou-se associação entre IU no terceiro trimestre gestacional com estado marital $(p=0,032)$, bronquite crônica $(p=0,011)$, depressão $(p=0,049) \mathrm{e}$ constipação antes da gestação $(p=0,013)$, de modo que as mulheres com IU tiveram maiores proporções de constipação antes da gravidez, de bronquite crônica e de depressão se comparadas às mulheres continentes (Tabela 1). Com relação ao estado marital, houve maior proporção de mulheres que viviam com seus parceiros no estrato incontinente. Os demais aspectos pesquisados - diabetes, asma, tabagismo, constipação no terceiro trimestre gestacional e atividade física antes da gestação e no terceiro trimestre gestacional - não apresentaram associação significativa com a IU no período investigado.

As mulheres incontinentes no terceiro trimestre eram significativamente mais velhas que as continentes (Tabela 2). Em relação aos dados obstétricos, houve diferença significativa entre mulheres continentes e incontinentes quanto ao número de gestações, sendo que as mulheres incontinentes haviam tido, de modo geral, maior número de gestações se comparadas às continentes. Além disso, as mulheres incontinentes tiveram mais queixas de perda urinária (maior frequência e maior intensidade) antes da gestação.

\section{Discussão}

O presente estudo identificou um aumento da prevalência de IU de $9,5 \%$ antes da gestação para $59,5 \%$ no terceiro trimestre gestacional. Estudo com gestantes brasileiras em São Paulo também identificou alta prevalência de sintomas no trato urinário inferior $(63,3 \%) .13$ Prevalências similares (antes da gestação e nas últimas semanas da gestação) foram reportadas em estudos na Austrália com mulheres nulíparas ${ }^{14}$ e na Noruega em estudo de base populacional. ${ }^{2}$ Esses dados demonstram o impacto da gestação avançada no aumento da IU. Kocaöz et al. ${ }^{6}$ realizaram na Turquia uma pesquisa semelhante utilizando o ICIQ-SF e verificaram prevalência de incontinência urinária bem inferior $(27,0 \%)$, embora não tenham controlado o trimestre gestacional.

Existe uma gama de recursos disponíveis e em expansão para prevenção de IU, incluindo controle do peso corporal, prática de exercícios do assoalho pélvico e mudança de hábitos, como melhorar o funcionamento intestinal e parar de fumar. ${ }^{15}$ Mesmo assim, a prevalência de IU durante a gestação ainda foi elevada neste estudo. Tal achado remete à pouca importância atribuída ao fator preventivo na atual conjuntura das ações em saúde, nas quais a maior parte da população desconhece as possibilidades de 
prevenção e controle.

Assim como em outros estudos, $, 2,6$ a maioria das gestantes apresentou vazamento de menos de uma vez por semana e em gotas antes e durante a gravidez. As situações mais comuns de perda urinárias entre as mulheres deste estudo incluíram ocasiões de esforço. Segundo Brown et al.,14 a incontinência de esforço e a incontinência mista foram mais comuns durante a gravidez do que a incontinência de urgência isoladamente. $\mathrm{O}$ acentuado aumento de peso corporal figura como principal fator contribuinte para o surgimento da IU de esforço nesse período. 6

Neste estudo, muitas das mulheres com sintomas de perdas urinárias $(25,7 \%)$ referiram que tal condição não interfere na qualidade de vida, o que vem ao encontro de outros estudos cujas participantes encaram as perdas como sintoma normal da gestação. 6 Mesmo que as mulheres não atribuam grande importância às perdas urinárias durante a gestação, estas parecem ter impacto futuramente, já que as mulheres que sofrem desse quadro durante a gestação apresentam risco aumentado para desenvolver IU após o parto. 16

Observou-se que as mulheres que apresentaram IU na gravidez eram mais velhas que as mulheres continentes, haja vista o processo natural de envelhecimento somado às compressões mecânicas do ciclo gravídico. Outros estudos identificaram que a idade materna é um fator de risco para IU gestacional.2,6,16 No entanto, de acordo com a revisão de Hijaz et al.,17 existem evidências contraditórias de que a idade materna avançada seja um fator de risco para a IU pós-gestacional, sendo necessários mais estudos para esclarecer tal aspecto.

Além disso, as mulheres que apresentaram IU na gravidez haviam tido mais gestações que as mulheres sem queixas de IU. A paridade foi identificada como um fator de risco para IU em análises ajustadas tanto antes da gravidez quanto durante esse período. Essa temática foi bastante explorada, mas ainda requer investigações que considerem outras variáveis clínicas, comportamentais e sociodemográficas. $2,6,10,13$

Neste estudo, a bronquite esteve associada à IU no terceiro trimestre gestacional. Segundo Martins et $a l ., 18$ a bronquite é identificada como um fator de risco para a falha do tratamento cirúrgico para correção de IU, assim como no processo de reabilitação a médio e longo prazo. Uma explicação para tal achado é a tosse, sintoma predominante dessa afecção, a qual está diretamente relacionada à incontinência de esforço, cujas manifestações clínicas incluem perda involuntária de urina durante situ- ações que caracterizam o esforço físico causado pelo aumento da pressão intra-abdominal. 19

Outra condição encontrada neste estudo como associada à IU foi a constipação antes da gestação, o que também foi verificado em outros estudos recentes.6,13 A constipação intestinal é foco de atenção em relação à $\mathrm{IU}$, visto que a presença de impactação fecal pode, muitas vezes, ser a causa de perdas urinárias, e a simples normalização desse problema contribui para a melhora dos sintomas de IU.19,20 Ainda não se sabe se a constipação crônica, assim como as causas obstétricas (paridade, partos vaginais, uso de fórceps e episiotomia), pode causar desenervação do nervo pudendo. Porém, já foi relatada associação entre constipação e lesões do assoalho pélvico em análises multivariadas que consideram tais variáveis obstétricas. 12

Além desses fatores, verificou-se que a perda de urina antes da gestação esteve associada a perdas de urina no último trimestre gestacional, indicando que mulheres com histórico de IU antes da gestação têm maior probabilidade de apresentar essa condição com mais intensidade no último trimestre, achado já constatado em outros estudos. $6,13,14$

Gestantes que relataram episódios depressivos durante a gestação apresentaram maiores proporções de IU nesse período que as gestantes sem tais sintomas. Nesse sentido, um estudo de base populacional com mulheres não gestantes identificou que a depressão foi capaz de predizer o início da IU.21 Portanto, são necessários estudos clínicos que avaliem a relação entre alterações emocionais e desencadeamento de episódios de perdas urinárias.

Nossos resultados demonstram maior proporção de mulheres que vivem com seus parceiros no estrato incontinente em comparação ao continente. A análise utilizada neste estudo, em que o possível fator de confusão idade não foi considerado, limitou a interpretação desse resultado.

Assim, a identificação dos referidos fatores associados pode contribuir com a assistência à saúde da mulher e a adoção de intervenções para prevenção, diagnóstico e tratamento relacionados à perda urinária tanto na gestação quanto em todo o ciclo de vida da mulher.

No presente estudo, identificou-se alta prevalência de incontinência urinária no terceiro trimestre gestacional $(59,5 \%)$ em uma maternidade pública de referência no Estado de Santa Catarina. Também foram identificadas algumas variáveis associadas à IU na gestação, incluindo idade, número de gestações, estado marital (viver com o parceiro), constipação antes da gravidez, bronquite crônica e sintomas depressivos. 
Este estudo limita-se por avaliar a IU gestacional em desenho de estudo transversal e por utilizar um questionário validado, porém baseado no autorrelato, para mensurar as perdas urinárias. Sugere-se a rea-

\section{Referencias}

1. Santos PC, Mendonça D, Alves O, Barbosa AM. Prevalência e impacto da incontinência urinária de estresse antes e durante a gravidez. Acta Med Port. 2006; 19: 34956.

2. Wesnes SL, Rortveit G, Bø K, Hunskaar S. Urinary incontinence during pregnancy. Obstet Gynecol. 2007; 109 (4) 922-8.

3. Oliveira E, Zuliani LMM, Ishicava J, Silva SV, Albuquerque SSR, Souza AMB, Barbosa CP. Avaliação dos fatores relacionados à ocorrência da incontinência urinária feminina. Rev. Assoc Med Bras. 2010; 56 (6): 688-90.

4. Figueiredo, EM, Lara, JO, Cruz, MC, Quintão, DMG, Monteiro, MVC. Perfil sociodemográfico e clínico de usuárias de serviço de fisioterapia uroginecológica da rede pública. Rev Bras Fisioter. 2008; 12 (2): 136-42.

5. Bø K, Owe KM, Nystaad W. Which women do pelvic floor muscle exercises six months' postpartum? Am J Obstet Gynecol. 2007; 197 (1): 49-5.

6. Kocaöz S, Talas MS, Atabekoğlu CS. Urinary incontinence in pregnant women and their quality of life. J Clin Nurs 2010; 19 (23-24): 3314-23.

7. Lopes DBM, Praça NS. Incontinência urinária autorreferida no pós-parto. Texto Contexto Enferm. 2010; 19(4): 667-74.

8. Zanetti MRD, Castro RA, Rotta AL, Santos PD, Sartori M, Girão MJBC. Impact of supervised physiotherapeutic pelvic floor exercises for treating female stress urinary incontinence. São Paulo Med J. 2007; 125 (5): 265-9.

9. Scarpa KP, Herrmann V, Palma PCR, Ricetto CLZ, Morais S. Sintomas do trato urinário inferior três anos após o parto estudo prospectivo. Rev Bras Ginecol Obstet. 2008; 30 (7): 355-9.

10. Higa R, Lopes MHM, Reis MJ. Fatores de risco para incontinência urinária na mulher. Rev Esc Enferm USP. 2008; 42 (1): $187-92$

11. Tamanini JTN, Damdros M, D'ancora CAL, Palma PCR, Rodrigues Netto Jr N. Validação para o português do "International Consultation on Incontinence Questionnaire - Short Form” (ICIQ-SF). Rev Saúde Pública. 2004; 38 (3) 438-44.

Recebido em 4 de dezembro de 2012

Versão final apresentada em 12 de junho de 2013

Aprovado em 12 de julho de 2013 lização de estudos de coorte levando-se em consideração a mulher desde o estado pré-gravídico até o puerperal com um rigoroso controle das variáveis clínicas, obstétricas e sociodemográficas.

12. Amselem C, Puigdollers A, Azpiroz F, Sala C, Videla S, Fernández-Fraga $\mathrm{X}$, Whorwell $\mathrm{P}$, Malagelada JR. Constipation: a potential cause of pelvic floor damage? Neurogastroenterol Motil. 2010; 22: 150-e48.

13. Martins G, Soler ZSG, Cordeiro JA, Amaro JL, Moore KN. Prevalence and risk factors for urinary incontinence in healthy pregnant Brazilian women. Int Urogynecol J. 2010; 21 (10): 1271-7.

14. Brown SJ, Donath S, MacArthur C, McDonald E, Krastev $\mathrm{AH}$. Urinary incontinence in nulliparous women before and during pregnancy: prevalence, incidence, and associated risk factors. Int Urogynecol J. 2010; 21 (2): 193-202.

15. Herbruck LF. Stress urinary incontinence: prevention, management, and provider education. Urologic Nur. 2008; 28 (3): 200-7.

16. Solans-Domènech M, Sánchez E, Espuña-Pons M. Urinary and anal incontinence during pregnancy and postpartum: incidence, severity, and risk factors. Obstet Gynecol. 2010; 115 (3): 618-28.

17. Hijaz A, Sadeghi Z, Byrne L, Hou JCT, Daneshgari F. Advanced maternal age as a risk factor for stress urinary incontinence: a review of the literature. Int Urogynecol J. 2012; 23: 395-401

18. Martins JAM, Castro RA, Girão MJB, Sartori MGF, Baracat EC, Lima GR. Correção da incontinência urinária de esforço com sling: resultados iniciais. Rev Bras Ginecol Obstet. 2000; 22 (5): 301-5.

19. Callahan TL, Caughey AB. Ginecologia e obstetrícia. 4 ed. Rio de Janeiro: Revinter; 2010.

20. Burgio KL, Mathews KA, Engel, BT. Prevalence, incidence and correlates of urinary incontinence in healthy, middleaged women. J Urol. 1991; 146: 1255-9.

21. Melville JL, Fan MY, Rau H, Nygaard IE, Katon WJ. Major depression and urinary incontinence in women: temporal associations in an epidemiologic sample. Am J Obstet Gynecol. 2009; 490: e2-e7. 